\title{
Predicting retinal tissue oxygenation using an image-based theoretical model
}

\author{
Brendan C. Fry ${ }^{\mathrm{a}}$, Ehren Brant Coburn ${ }^{\mathrm{b}}$, Spencer Whiteman ${ }^{\mathrm{b}}$, \\ Alon Harris ${ }^{c}$, Brent Sieskyc ${ }^{c}$, Julia Arciero ${ }^{b}$
}

aDepartment of Mathematical and Computer Sciences, Metropolitan State University of Denver, P.O. Box 173362, Campus Box 38, Denver, CO 80217, USA

bDepartment of Mathematical Sciences, Indiana University-Purdue UniversityIndianapolis, 402 N. Blackford St, LD 270, Indianapolis, IN 46202, USA

'Department of Ophthalmology, Glick Eye Institute, Indiana University School of Medicine, 1160 W. Michigan St., Indianapolis, IN 46202, USA

Brendan C. Fry: bfry2@msudenver.edu Ehren Brant Coburn: ecoburn@iupui.edu Spencer Whiteman: slwhitem@imail.iu.edu Alon Harris: alharris@indiana.edu Brent Siesky: bsiesky@indiana.edu Julia Arciero: jarciero@iupui.edu

Corresponding Author: Brendan C. Fry, bfry2@msudenver.edu

\begin{abstract}
Impaired oxygen delivery and tissue perfusion have been identified as significant factors that contribute to the loss of retinal ganglion cells in glaucoma patients. This study predicts retinal blood and tissue oxygenation using a theoretical model of the retinal vasculature based on confocal microscopy images of the mouse retina. These images reveal a complex and heterogeneous geometry of vessels that are distributed nonuniformly into multiple distinct retinal layers at varying depths. Predicting oxygen delivery and distribution in this irregular arrangement of retinal microvessels requires the use of an efficient theoretical model. The model employed in this work utilizes numerical methods based on a Green's function approach to simulate the spatial distribution of oxygen levels in a network of retinal blood vessels and the tissue surrounding them. Model simulations also predict the blood flow rates and pressures in each of the microvessels throughout the entire network. As expected, the model predicts that average vessel $\mathrm{PO}_{2}$ decreases as oxygen demand is increased. However, the standard deviation of $\mathrm{PO}_{2}$ in the vessels nearly doubles as oxygen demand is increased from 1 to $8 \mathrm{~cm}^{3} \mathrm{O}_{2} / 100 \mathrm{~cm}^{3} / \mathrm{min}$, indicating a very wide spread in the predicted $\mathrm{PO}_{2}$ levels, suggesting that average $\mathrm{PO}_{2}$ is not a sufficient indicator of oxygenation in a heterogeneous vascular network. Ultimately, the development of this mathematical model will help to elucidate the important factors associated with blood flow and metabolism that contribute to the vision loss characteristic of glaucoma.
\end{abstract}




\section{Highlights}

- Retinal blood and tissue oxygenation is modeled in a heterogeneous vascular network.

- The model uses a numerical method based on Green's functions.

- Simulations also predict blood ow rates and pressures in each microvessel.

- As $\mathrm{O}_{2}$ demand increases, mean $\mathrm{PO}_{2}$ decreases and standard deviation of $\mathrm{PO}_{2}$ increases.

- At high $\mathrm{O}_{2}$ demand, mean $\mathrm{PO}_{2}$ is a poor oxygenation indicator in heterogeneous net-works.

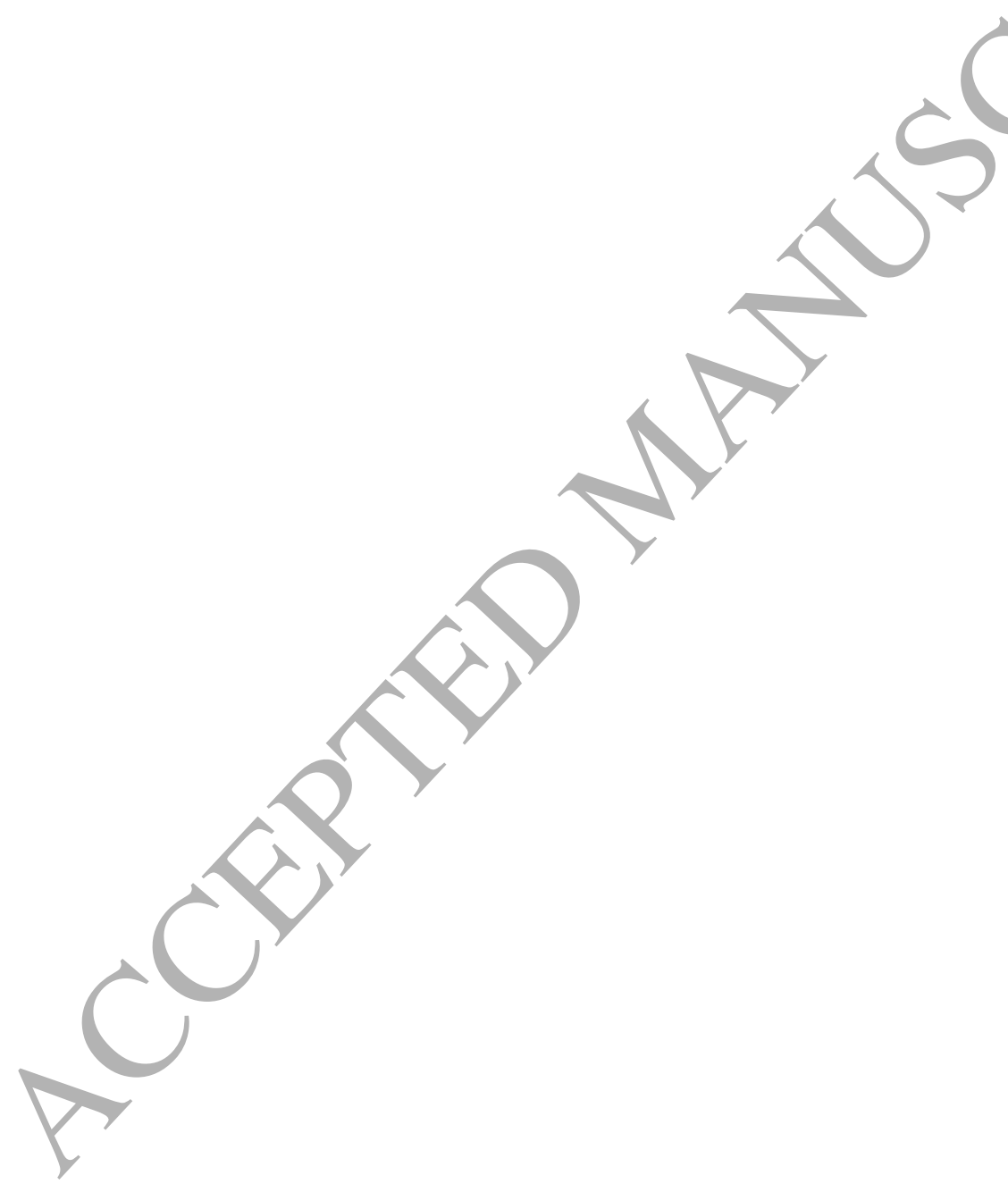




\section{Keywords}

Mathematical model, retina, oxygenation, Green's functions, microcirculation, glaucoma

\section{Introduction}

Primary open angle glaucoma (POAG) is the second-leading cause of blindness worldwide and is characterized by progressive retinal ganglion cell death and vision loss. The only approved treatments for POAG are interventions aimed at lowering intraocular pressure (IOP), which is an established risk factor for POAG. However, at least one-third of glaucoma patients do not exhibit elevated IOP yet still progress to advanced stages of glaucoma [1,2]. Additionally, several studies [3-6] have shown that impaired oxygen delivery and tissue perfusion are also significant factors that contribute to the destruction of retinal ganglion cells. It is unknown, however, whether changes in blood flow to the retina are the cause or effect of retinal ganglion cell death. Retinal blood flow that does not meet the oxygen demand of the tissue can result in regions of tissue ischemia and retinal ganglion cell destruction. This would indicate a significant role for blood flow in the pathophysiology of glaucoma. Conversely, the death of retinal ganglion cells can lead to a decrease in metabolic demand for oxygen or nutrients and would indicate that impaired or reduced blood flow is a result of glaucoma. The current work provides a first step toward addressing this controversy by predicting tissue oxygen saturation in a realistic vascular network geometry of the retina.

In the clinic, blood oxygen saturation levels and diameters are obtained for retinal arterioles and venules both inferior and superior to the optic nerve head via noninvasive spectrophotometric retinal oximetry methods. In healthy individuals, blood oxygen saturation is typically $90-100 \%$ in arterioles and $50-75 \%$ in venules. Recently, Olafsdottir et al. [7] found that venous oxygen saturation was higher in patients with advanced glaucoma than in healthy individuals. They concluded that this was an indication of lower oxygen demand in advanced glaucomatous patients, suggesting that the abnormal oxygen metabolism was the result of glaucomatous damage. However, as shown in a recent theoretical study [8], several other factors can lead to increased venous saturation, including impaired blood flow autoregulation mechanisms and decreased tissue volume. This suggests that oxygen saturation data obtained from oximetry maps are not sufficient to identify the mechanisms that cause alterations in venous oxygen saturation. Instead, combined theoretical methods and clinical measures are needed to identify the cause-and-effect relationships between retinal function, blood flow, oxygen levels, and IOP.

Previous theoretical models have been developed to predict retinal blood flow and/or oxygenation within various retinal networks. For example, Bernabeu et al. [9] developed a three-dimensional reconstruction of murine retinal arteries based on confocal microscopy images and predicted velocity and shear stress throughout the vasculature within five to six days of birth. The model estimates hemodynamic changes during vascular remodeling and regression, and thus has important implications for angiogenesis studies. Liu et al. [10] developed a quantitative model of flow and oxygen transport in the retina to assess the impact of topological changes in the retinal vasculature on oxygen distribution. The model couples the Navier-Stokes equations to 
convection-diffusion equations for oxygen transfer to tissue to predict flow and oxygenation. The model is simulated within a realistic depiction of the central retinal artery and retinal arteriolar branches (obtained from fundus images in human) and a virtual depiction of peripheral vessels. The model is used to predict oxygen saturation in arterioles, but does not include venous saturation predictions or any aspects of flow regulation. Causin et al. [11] developed a 6-layer model (3 layers for the inner retina and 3 layers for the outer retina) that couples blood flow mechanics to a vascular network model for oxygen distribution in tissue. The vascular network model consists of arterioles and venules represented as discrete fractal trees and a capillary plexus represented as a set of parallel pipes. Within the context of this network, they predict the effects of changes in blood pressure, blood rheology (e.g., plasma viscosity and metabolic consumption rates), and arterial oxygen permeability on tissue and blood oxygen saturation. Such a comprehensive study offers a great deal of insight into retinal hemodynamics, although the network structure is not based on an experimentally observed structure. Malek et al. [12] used a fundus image from a healthy human adult to create a network model for the large arteries and veins of the retinal vasculature. For smaller vessels that are indistinguishable in the fundus image, a fractal structured tree is implemented. Blood flow is described using the Navier-Stokes equations, and the velocity and pressure profiles along the network are predicted. Blood and tissue oxygenation, however, are not predicted with this model. This model was later used to study the effect of topological changes (such as tortuosity) on hemodynamics in retinal circulation [13]. Ganesan et al. [14, 15] presented an image-based network model of the murine retinal vasculature using confocal microscopy images. The capillaries are represented using a rule-based mesh, and model simulations are performed to predict the spatial distribution of pressure, flow, and wall shear stress in the entire retinal vasculature, but no prediction of blood or tissue oxygenation are obtained in their model.

Theoretical models capable of simulating flow and oxygenation in realistic, heterogeneous vascular networks have been developed in multiple tissues other than the retina [16-18]. For example, Secomb et al. have developed strategies for predicting oxygen transport in a heterogeneous network using techniques from potential theory involving Green's functions and have applied them to microvascular networks in the mesentery, in striated muscle, and in tumors $[16,17]$. Concurrently, imaging studies in the coronary microvasculature coupled with improved computational techniques have allowed for prediction of blood flow rates in large networks [19-22], and progress is being made in using in vivo imaging of the cerebral vasculature to predict flows and oxygenation in the microcirculation of the brain [23].

The present study builds upon aspects of many of these previous approaches with the ultimate goal of predicting tissue oxygen levels (in addition to pressure, flow, velocity, and viscosity) within a realistic, heterogeneous retinal vascular network geometry obtained from confocal microscopy images [15]. To simulate the spatial distribution of oxygen levels in the blood vessels and tissue surrounding a network of retinal blood vessels, numerical methods based on a Green's function approach are adapted to the 
retina. Simulations are conducted for varying levels of oxygen demand, and the impact of a spatially heterogeneous network model on tissue oxygenation is demonstrated.

\section{Material and Methods}

\section{$2.1 \quad$ Network geometry}

To simulate oxygen transport in a realistic microvascular network of the retina, confocal microscopy images of arterioles and venules and a rule-based capillary model presented by Ganesan et al. [14, 15] are adapted to define the connections between retinal arterioles, capillaries, and venules in the present study. Several network quantities, including arteriolar and venular diameters, capillary density, capillary ring radius, capillary length, and number of interconnecting vessels, are taken directly from Ganesan et al. [14, 15].

The composition of the retinal vasculature must be understood to generate an accurate heterogeneous vascular network model in this study. The retina is divided into ten distinct layers [24], of which only three of these layers are vascularized. Blood is supplied to the retina via the central retinal artery, which gives rise to the main branches of the retinal circulation. The retinal arteries travel outwards toward the nerve fiber layer to feed two types of capillary systems (capillaries that supply the superficial nerve layer and deep branches that supply the inner retina). Retinal blood is returned via retinal venules that drain into the central retinal vein. In the study by Ganesan et al. [15], the confocal microscopy images reveal this complex geometry of vessels which are distributed non-uniformly into three distinct retinal layers at varying depths: the superficial layer (SL), intermediate layer (IL), and deep layer (DL) (Figure 1). In the model, the superficial layer is assumed to contain all of the arterioles and large venules (approximate diameter of $20 \mu \mathrm{m}$ or larger). The intermediate layer contains only capillaries, and the deep layer includes capillaries and the remaining (smaller) venules. In the model, the intermediate layer is defined $55 \mu \mathrm{m}$ below the superficial layer, and the deep layer is defined $50 \mu \mathrm{m}$ below the intermediate layer (Figure 1).

Vessels in the three layers are connected via six different connection types, as proposed by Ganesan et al. [14, 15]. These connections originate or terminate with a terminal vessel, which is defined as an arteriole or venule that has only one vessel attached to it (see Figure 1). As outlined in Table 1, terminal arterioles are connected to a terminal venule in the superficial layer via a Type 1 connection. The remaining terminal arterioles are connected to one capillary in the intermediate layer (Type 3) and three capillaries in the deep layer (Type 2). Similarly, terminal venules in the deep layer are connected to one capillary in the intermediate layer (Type 5) and three capillaries in the deep layer (Type 6). The remaining interconnecting vessel capillaries in the intermediate layer are connected to one capillary in the deep layer (Type 4). Multiple connections from a single terminal vessel are not permitted for Types 1 and 4 . All connections are made using a minimum distance algorithm that calculates the minimum distance between the endpoints of vessels (defined herein as nodes). 

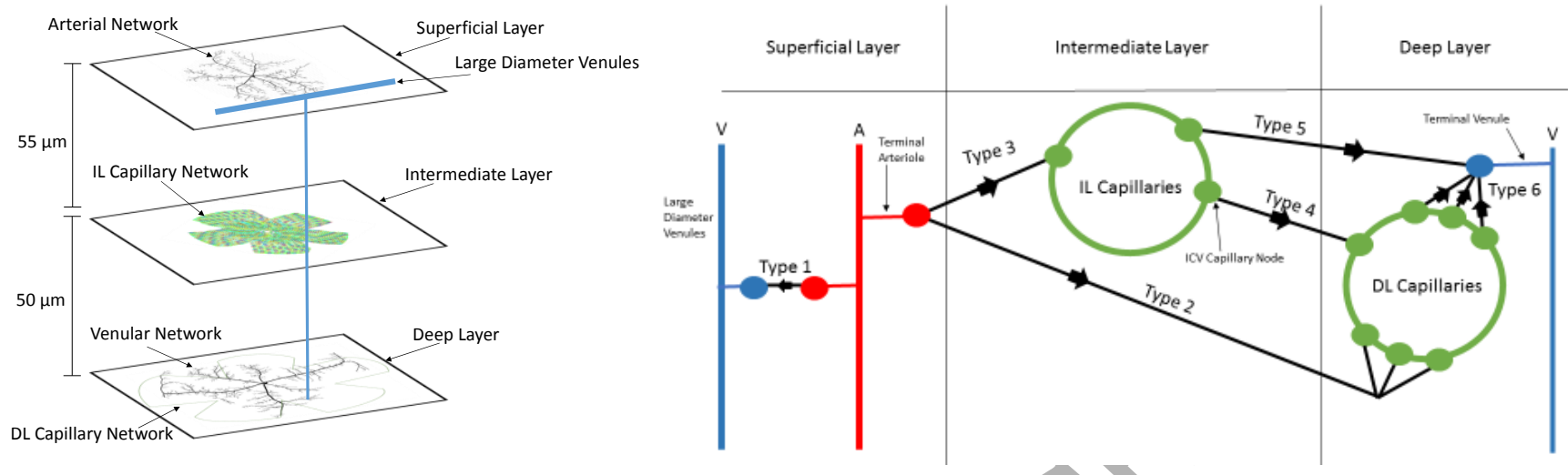

Figure 1. (Left) Schematic of the three retinal layers: superficial, intermediate, and deep layers. (Right) Schematic illustrating the six connection types among vessels.

\begin{tabular}{|c|c|c|c|}
\hline Type & Nodes Used & $\begin{array}{c}\text { Number of } \\
\text { Segments }\end{array}$ & Diameter \\
\hline 1 & Terminal Arteriole $\rightarrow$ Terminal Venule in SL & 18 & 5.75 \\
\hline 2 & Terminal Arteriole $\rightarrow$ 3 DL Capillaries & $642^{*} 3=1926$ & 5.75 \\
\hline 3 & Terminal Arteriole $\rightarrow$ 1 IL Capillary & 642 & 5.75 \\
\hline 4 & Unused IL ICV Capillary $\rightarrow$ 1 DL Capillary & 990 & 5 \\
\hline 5 & 1 IL Capillary $\rightarrow$ Terminal Venule in DL & 1073 & 5 \\
\hline 6 & 3 DL Capillaries $\rightarrow$ Terminal Venule in DL & $1073^{\star} 3=3219$ & 5 \\
\hline
\end{tabular}

Table 1. Six types of vascular connections among the three layers

\subsubsection{Arterioles and Venules}

As established by Ganesan et al. [14, 15], the arteriolar and venular networks of the mouse retina have 6 main branches that are fed and drained by the central retinal artery and central retinal vein, respectively. The positions and diameters of the arteriolar and venular networks obtained by Ganesan et al. are used directly in this study, although the quantities of each vessel type differ slightly in the present work from the values obtained by Ganesan et al. due to slight differences in the classification of vessel order (see Table 2). The arteriolar and venular networks used in the current model are depicted in Figure 2. 


\begin{tabular}{|c|c|c|c|}
\hline Network & Order & Diameter $(\mu \mathrm{m})$ & Quantity \\
\hline \multirow[t]{5}{*}{ Artery 1} & 1 & 5.75 & 61 \\
\hline & 2 & 7.71 & 32 \\
\hline & 3 & 10.28 & 15 \\
\hline & 4 & 14.42 & 6 \\
\hline & 5 & 21.16 & 4 \\
\hline \multirow[t]{5}{*}{ Artery 2} & 1 & 5.75 & 221 \\
\hline & 2 & 7.94 & 75 \\
\hline & 3 & 11.54 & 25 \\
\hline & 4 & 18.86 & 10 \\
\hline & 5 & 30.42 & 7 \\
\hline \multirow[t]{5}{*}{ Artery 3} & 1 & 5.75 & 136 \\
\hline & 2 & 7.87 & 48 \\
\hline & 3 & 12.18 & 12 \\
\hline & 4 & 17.98 & 9 \\
\hline & 5 & 26.84 & 6 \\
\hline \multirow[t]{4}{*}{ Artery 4} & 1 & 5.75 & 126 \\
\hline & 2 & 7.79 & 39 \\
\hline & 3 & 11.65 & 14 \\
\hline & 4 & 22.91 & 7 \\
\hline \multirow[t]{5}{*}{ Artery 5} & 1 & 5.75 & 102 \\
\hline & 2 & 8.03 & 48 \\
\hline & 3 & 11.47 & 12 \\
\hline & 4 & 16.01 & 9 \\
\hline & 5 & 24.02 & 6 \\
\hline \multirow[t]{5}{*}{ Artery 6} & 1 & 5.75 & 147 \\
\hline & 2 & 8.01 & 69 \\
\hline & 3 & 11.05 & 13 \\
\hline & 4 & 19.56 & 4 \\
\hline & 5 & 28.48 & 4 \\
\hline
\end{tabular}

\begin{tabular}{|c|c|c|c|}
\hline Network & Order & Diameter $(\mu \mathrm{m})$ & Quantity \\
\hline \multirow[t]{4}{*}{ Vein 1} & 1 & 5 & 61 \\
\hline & 2 & 7.1 & 38 \\
\hline & 3 & 10.71 & 6 \\
\hline & 4 & 17.22 & 14 \\
\hline \multirow[t]{4}{*}{ Vein 2} & 1 & 5 & 198 \\
\hline & 2 & 8.07 & 74 \\
\hline & 3 & 13.65 & 33 \\
\hline & 4 & 26.48 & 34 \\
\hline \multirow[t]{4}{*}{ Vein 3} & 1 & 5 & 149 \\
\hline & 2 & 7.09 & 48 \\
\hline & 3 & 10.41 & 11 \\
\hline & 4 & 20.27 & 18 \\
\hline \multirow[t]{5}{*}{ Vein 4} & 1 & 5 & 195 \\
\hline & 2 & 6.86 & 71 \\
\hline & 3 & 11.09 & 39 \\
\hline & 4 & 17.8 & 15 \\
\hline & 5 & 27.15 & 13 \\
\hline \multirow{5}{*}{ Vein 5} & 1 & 5 & 249 \\
\hline & 2 & 7.34 & 126 \\
\hline & 3 & 10.8 & 49 \\
\hline & 4 & 15 & 13 \\
\hline & 5 & 26.73 & 32 \\
\hline \multirow[t]{5}{*}{ Vein 6} & 1 & 5 & 234 \\
\hline & 2 & 7.69 & 94 \\
\hline & 3 & 11.06 & 53 \\
\hline & 4 & 16.08 & 8 \\
\hline & 5 & 29.42 & 15 \\
\hline
\end{tabular}

Table 2. Order number, diameter, and quantity of arterioles and venules 

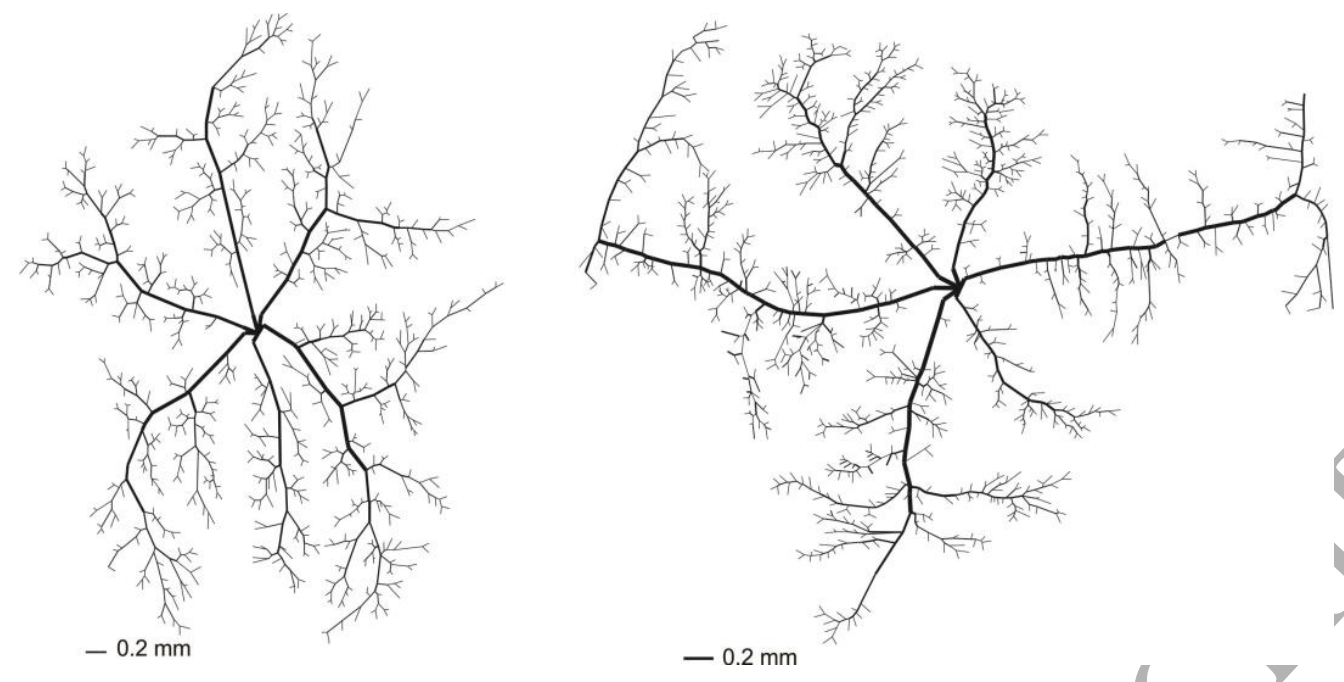

Figure 2. Depictions of arteriolar (left) and venular (right) networks.

\subsubsection{Capillaries}

Retinal capillaries are located in the intermediate and deep layers of the retina. In the model, each retinal layer is assumed to contain a mesh of concentric rings on which capillaries are evenly distributed, as proposed by Ganesan et al. [14]. Each ring of capillaries is connected to the next ring via capillaries termed interconnecting vessels (ICV) [14].

Using confocal microscopy images, Ganesan et al. $[14,15]$ determined that the innermost ring of capillaries should have a radius of $R=150 \mu \mathrm{m}$ and an outermost ring radius of $R=2750 \mu \mathrm{m}$ in both the intermediate and deep layers. Ganesan et al. also used the confocal microscopy images to quantify the number of interconnecting vessels that should be used to connect each capillary ring. Using the innermost ring radius and the number of interconnecting vessels (values taken directly from Ganesan et al. [14]), the remaining ring radii are calculated using a least squares algorithm to determine the capillary density (defined as the total length of capillaries per area of the tissue region) in the intermediate and deep layer that would minimize the error between the calculated and observed outermost ring radius. The calculation for the ring radius is given in Eq. 1:

$$
R_{k+1}=\frac{\left(2 \pi+I_{\text {ves }}\right)+\sqrt{\left(2 \pi+I_{\text {ves }}\right)^{2}-4 \pi(C D)\left(I_{\text {ves }} R_{k}-(C D) \pi R_{k}^{2}\right)}}{2 \pi(C D)}
$$

where $R_{k}$ is the radius of the $k^{\text {th }}$ ring, $I_{\text {ves }}$ is the number of interconnecting vessels between rings $R_{k}$ and $R_{k+1}$, and $C D$ is the capillary density. Here, the capillary density is determined to be $0.018 \mu \mathrm{m} / \mu \mathrm{m}^{2}$ for the intermediate layer and $0.024 \mu \mathrm{m} / \mu \mathrm{m}^{2}$ for the deep layer. Although similar, we note these density values differ slightly from those used by Ganesan et al. $\left(0.02 \mu \mathrm{m} / \mu \mathrm{m}^{2}\right.$ in the intermediate layer and $0.03 \mu \mathrm{m} / \mu \mathrm{m}^{2}$ in the deep layer) [15]. 
The number of capillaries comprising each ring is estimated from the distribution of capillary lengths at varying radial distance provided in $[14,15]$. More precisely, the number of capillary nodes on each ring, $n_{k}$, is calculated as:

$$
n_{k}=\frac{2 \pi R_{k}}{l_{k}}
$$

where $I_{k}$ is the average capillary length measurement obtained from the distribution of lengths measured by Ganesan et al. [14, 15]. The values $n_{k}$ are rounded to guarantee integer values for the number of capillary nodes on each ring. The nodes are arranged evenly on each ring, and the angle, $\theta_{k}$, between each node is given by

$$
\theta_{k}=\frac{2 \pi}{n_{k}} .
$$

Interconnecting vessels are assumed to be spaced evenly along each ring. Eq. 4 is used to calculate the distance $\left(n_{\text {space }}\right)$ along each ring between interconnecting vessels:

$$
n_{\text {space }}=\frac{n_{k}}{I_{\text {ves }}}
$$

This quantity, $n_{\text {space }}$, is rounded and used to determine which capillary nodes are also the initial nodes for interconnecting vessels. The terminal node for each interconnecting vessel is calculated to be the node on the next capillary ring that is the minimum distance from the initial node. Table 3 lists the number of capillary rings, ring radii, number of interconnecting vessels, and number of nodes on each ring for the intermediate and deep layers.

When the confocal microscopy images were obtained by Ganesan et al. [14, 15], the mouse retina was cut in five places to allow the eye to be laid flat for imaging. To maintain accuracy between the images and data provided by Ganesan et al. [14, 15], cuts were made in the capillary network in our model (see Fig 3). The capillary nodes in these five cut regions were relocated (not removed) based on a bisection rule. In particular, any node on the left side of the bisection was moved to the left of the cut, and any node on the right side of the bisection was moved to the right of the cut. Once all the nodes were relocated, all capillaries were re-spaced evenly in the resulting regions. Importantly, since some of the initial nodes of the interconnecting vessels were relocated due to the cut regions, the positions of the interconnecting vessels changed slightly. The minimum distance algorithm was re-implemented with the revised list of capillary node coordinates to finalize all capillary node positions.

The capillary model for the IL and DL contains a large number of nodes: 6375 and 12897 , respectively. To reduce computation time, "dummy" nodes are removed (as in Ganesan et al. [14]). Dummy nodes are defined as any capillary nodes that are only connected to two other nodes (i.e., they are not connected to an interconnecting vessel or any of the Type 1-6 connections). Flow calculations are not affected by removing such nodes, and computation time is significantly reduced. After removing the dummy nodes in the model, there are 3383 capillary nodes in the IL ( $47 \%$ reduction) and 8479 capillary nodes in the $\mathrm{DL}(\sim 34 \%$ reduction). 


\begin{tabular}{|c|c|c|c|c|c|c|}
\hline & \multicolumn{3}{|c|}{ Intermediate Layer } & \multicolumn{3}{|c|}{ Deep Layer } \\
\hline Ring \# & Radius ( $\mu \mathrm{m}$ ) & \# of Nodes & \# of ICV & Radius ( $\mu \mathrm{m}$ ) & \# of Nodes & \# of ICV \\
\hline 1 & 150.00 & 16 & 12 & 150.00 & 34 & 20 \\
\hline 2 & 292.37 & 35 & 14 & 291.74 & 92 & 24 \\
\hline 3 & 392.81 & 53 & 17 & 383.23 & 108 & 27 \\
\hline 4 & 486.88 & 56 & 19 & 463.85 & 124 & 31 \\
\hline 5 & 575.49 & 75 & 22 & 541.26 & 147 & 35 \\
\hline 6 & 662.78 & 74 & 24 & 616.75 & 152 & 39 \\
\hline 7 & 747.57 & 79 & 27 & 690.97 & 181 & 43 \\
\hline 8 & 831.99 & 91 & 29 & 764.30 & 213 & 47 \\
\hline 9 & 914.90 & 109 & 31 & 836.96 & 225 & 51 \\
\hline 10 & 996.64 & 110 & 34 & 909.11 & 254 & 55 \\
\hline 11 & 1078.46 & 110 & 36 & 980.85 & 278 & 59 \\
\hline 12 & 1159.42 & 128 & 39 & 1052.27 & 249 & 63 \\
\hline 13 & 1240.52 & 126 & 41 & 1123.43 & 281 & 67 \\
\hline 14 & 1320.95 & 137 & 43 & 1194.36 & 316 & 71 \\
\hline 15 & 1400.80 & 133 & 46 & 1265.11 & 344 & 75 \\
\hline 16 & 1480.86 & 131 & 45 & 1335.69 & 270 & 79 \\
\hline 17 & 1558.51 & 125 & 45 & 1406.15 & 256 & 82 \\
\hline 18 & 1634.73 & 124 & 45 & 1475.94 & 262 & 80 \\
\hline 19 & 1709.71 & 118 & 45 & 1542.77 & 260 & 80 \\
\hline 20 & 1783.60 & 120 & 45 & 1608.00 & 212 & 80 \\
\hline 21 & 1856.55 & 114 & 45 & 1671.85 & 204 & 80 \\
\hline 22 & 1928.64 & 108 & 45 & 1734.50 & 215 & 80 \\
\hline 23 & 1999.97 & 109 & 45 & 1796.10 & 254 & 80 \\
\hline 24 & 2070.62 & 115 & 45 & 1856.76 & 253 & 80 \\
\hline 25 & 2140.65 & 115 & 45 & 1916.58 & 206 & 80 \\
\hline 26 & 2210.11 & 113 & 45 & 1975.64 & 240 & 80 \\
\hline 27 & 2279.05 & 115 & 45 & 2034.02 & 216 & 80 \\
\hline 28 & 2347.51 & 112 & 45 & 2091.77 & 241 & 80 \\
\hline 29 & 2415.52 & 101 & 45 & 2148.94 & 244 & 80 \\
\hline 30 & 2483.13 & $\begin{array}{r}99 \\
\end{array}$ & 45 & 2205.59 & 200 & 80 \\
\hline 31 & 2550.36 & 98 & 45 & 2261.75 & 205 & 80 \\
\hline 32 & 2617.23 & 98 & 45 & 2317.46 & 220 & 80 \\
\hline 33 & 2683.77 & 91 & 45 & 2372.74 & 221 & 80 \\
\hline 34 & 2750.00 & 45 & 45 & 2427.64 & 218 & 80 \\
\hline 35 & 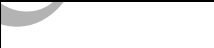 & & & 2482.16 & 212 & 80 \\
\hline 36 & & & & 2536.34 & 210 & 80 \\
\hline 37 & & & & 2590.20 & 185 & 80 \\
\hline 38 & & & & 2643.75 & 189 & 80 \\
\hline 39 & & & & 2697.01 & 187 & 80 \\
\hline 40 & & & & 2750.00 & 101 & 80 \\
\hline
\end{tabular}

Table 3. Radius, number of nodes, and number of interconnecting vessels on capillary rings in both IL and DL. 

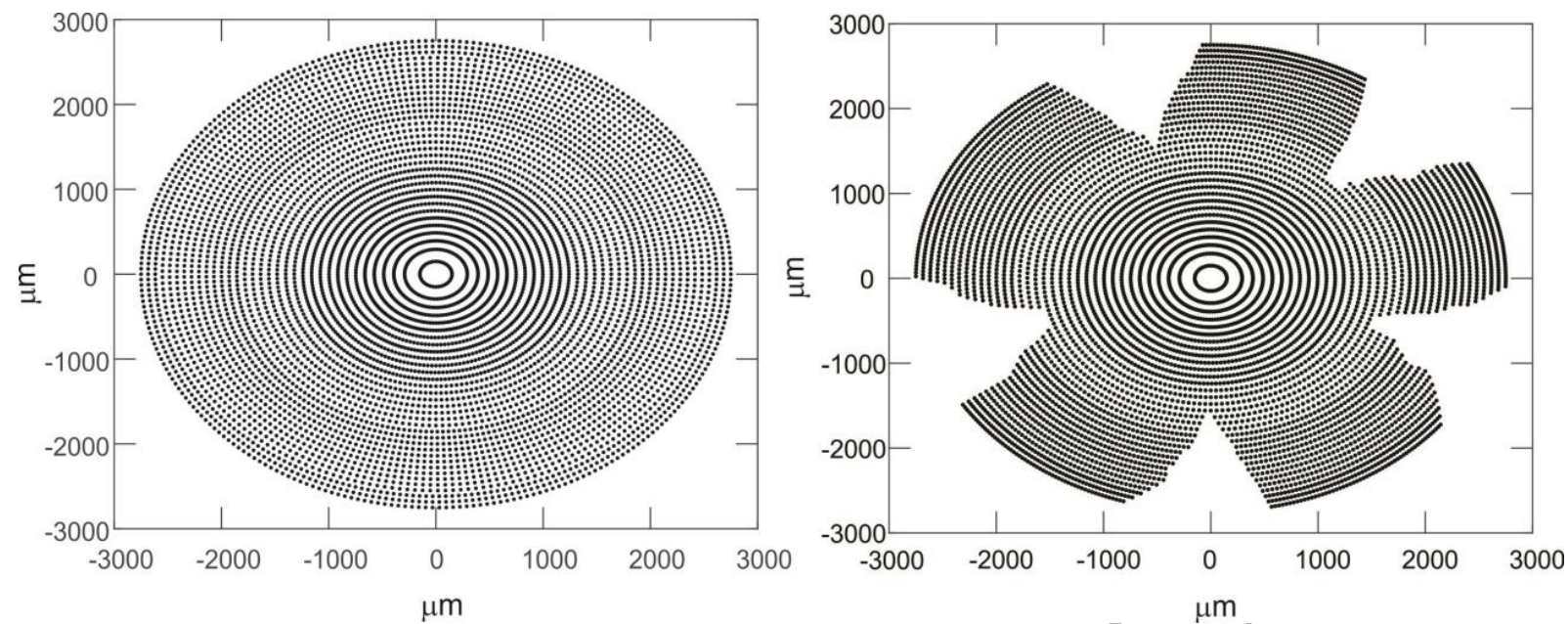

Figure 3. Capillary model without (left) and with (right) radial cuts for the intermediate layer (deep layer is similar, not shown).

\subsection{Hemodynamics in the network}

In this study, the microvascular network described in the previous section is represented as a directed graph, where each edge of the graph corresponds to a blood vessel segment. Each segment has a corresponding diameter and length; flow through each vessel is assumed to be pressure-driven following Poiseuille's law:

$$
Q=\Delta P \frac{\pi D^{4}}{128 \mu \mathrm{L}}
$$

where $Q$ is the flow rate in an individual vessel, $\Delta P$ is the pressure drop across the vessel, $D$ and $L$ are the diameter and length of the vessel, respectively, and $\mu$ is the apparent viscosity, which is assumed to depend on vessel diameter and hematocrit $\left(\mathrm{H}_{\mathrm{D}}\right)$, as described in $[25,26]$ :

where

$$
\mu=\left[1+\left(\mu_{0.45}-1\right) \frac{\left(1-H_{D}\right)^{C}-1}{(1-0.45)^{C}-1}\left(\frac{D}{D-1.1}\right)^{2}\right]\left(\frac{D}{D-1.1}\right)^{2}
$$

$$
\mu_{0.45}=6 e^{-0.085 D}+3.2-2.44 e^{-0.06 D^{0.645}}
$$

and

$$
C=\left(0.8+e^{-0.075 D}\right)\left(-1+\frac{1}{1+10^{-11} D^{12}}\right)+\frac{1}{1+10^{-11} D^{12}}
$$

To predict flows and pressures in a large heterogeneous network, an iterative technique (such as successive over-relaxation [27]) is necessary because the hematocrit (i.e., the red blood cell volume fraction) and the apparent viscosity are not constant among vessels. These two values and the flow rate in each vessel are interdependent. As a 
result, successive over-relaxation is used to make sequential calculations of the blood flow rate, hematocrit, and apparent viscosity in each of the vessel segments in the network.

To predict flows and pressures in a large heterogeneous network, an iterative technique is necessary because the hematocrit (i.e., the red blood cell volume fraction) and the apparent viscosity are not constant among vessels. In fact, these two values and the flow rate in each vessel are interdependent. Thus, flows, pressures, hematocrits, and apparent viscosities are calculated for each vessel segment of the network using a combination of two schemes [26]. In the first scheme, flow rates and pressures are initially calculated in each vessel segment of the network assuming a constant viscosity in all vessels. The inflow and outflow pressure or flow rate for the network are specified, and the conservation of flow at each node (where multiple vessel segments meet) is assumed. This yields a system of linear equations, where the unknowns are the nodal pressures. This large, sparse system can be solved using an iterative technique, such as successive over-relaxation [27].

The second scheme involves calculating the hematocrit and apparent viscosity in each segment, using the calculated flow rates and pressures from the first scheme. To calculate the hematocrit in each vessel, conservation of red blood cell flow rate and plasma flow rate is assumed at each node. In addition, an empirical relationship is used [26] describing the hematocrit of two daughter vessels in terms of the hematocrit of the parent vessel and the vessel diameters and flow rates. Since the hematocrit of a vessel is dependent on the upstream vessels in the network, the nodal pressures of the network are first partially sorted so that any two connected nodes appear in order of decreasing pressure. The nodes are then examined from largest to smallest pressure along each pathway. Once hematocrit is calculated in each vessel, Eqs. 6-8 [26] are used to calculate the apparent viscosity in each vessel. The two schemes are then alternately applied until sufficient convergence is reached.

\subsection{Green's function model for oxygen transport}

Several previous models of flow regulation in the retina and other tissues [28-32] have described tissue oxygenation using a Krogh cylinder model approach [33], which assumes that each vessel supplies oxygen only to a tissue cylinder immediately surrounding that vessel. This simplified model for oxygen exchange does not take into account diffusion from multiple vessels to one area of tissue and thus may underestimate the degree of hypoxia in tissue supplied by a heterogeneous microvascular network. In the present study, a more realistic model of oxygen transport is adopted which explicitly represents the interactions among vessels and tissue in a vascular network with non-uniform geometry.

The equation governing oxygen diffusion in the tissue is given by

$$
\operatorname{DaV}^{2} P_{O 2}=M
$$


where $D$ is the diffusion coefficient, $\alpha$ is the solubility of oxygen in tissue, and $M=M\left(P_{O 2}\right)$ is the oxygen consumption rate in the tissue that depends on the local tissue partial pressure of oxygen, $\mathrm{PO}_{2}\left(P_{O 2}\right)$. It is assumed that tissue oxygen consumption follows Michaelis-Menten kinetics, so that

$$
M\left(P_{O 2}\right)=M_{0} \frac{P_{O 2}}{P_{0}+P_{O 2}}
$$

where $M_{0}$ is the tissue oxygen demand, and $P_{0}$ is the $\mathrm{PO}_{2}$ at which the consumption is half maximal. Here, Da is fixed at $6 \times 10^{-10} \mathrm{~cm}^{3} \mathrm{O}_{2} / \mathrm{cm} / \mathrm{s} / \mathrm{mmHg}$ and $\mathrm{P}_{0}=10 \mathrm{mmHg}$, while $M_{0}$ is varied between 1 and $8 \mathrm{~cm}^{3} \mathrm{O}_{2} / 100 \mathrm{~cm}^{3} / \mathrm{min}$.

Due to the steep oxygen gradients near each of the vessels, predicting oxygen delivery and distribution in an irregular arrangement of retinal microvessels requires the use of an efficient theoretical model. The approach presented here utilizes techniques from potential theory, which seek to reduce the number of unknowns needed to represent the oxygen field. In this method, developed previously by Secomb et al. [16, 17] (referred to as the "Green's function approach"), vessels are considered discrete oxygen sources, and the tissue regions are considered oxygen sinks. To determine the source and sink strengths, it is assumed that the oxygen flux and $\mathrm{PO}_{2}$ at the blood-tissue interface are continuous. By conservation of mass,

$$
\frac{d f\left(P_{b}\right)}{d s}=-q_{v}(s)
$$

where $s$ is the length along a vessel segment, $q_{v}(s)$ is the rate of diffusive $\mathrm{O}_{2}$ efflux per unit vessel length, and $f\left(P_{b}\right)$ is the rate of convective $\mathrm{O}_{2}$ transport along a vessel segment. Thus, the source strength of a given vessel segment is strongly dependent on $f\left(P_{b}\right)$, which is determined by of a number of factors that make up the vascular phenotype - in particular the blood flow rate $(Q$, which is dependent on vessel diameter, length, and apparent blood viscosity) and hematocrit $\left(\mathrm{H}_{\mathrm{D}}\right)$ :

$$
f\left(P_{b}\right)=Q\left(H_{D} C_{0} S\left(P_{b}\right)+\alpha_{b} P_{b}\right)
$$

where $\mathrm{C}_{0}$ is the hemoglobin-bound oxygen concentration in a fully-saturated red blood cell, $S\left(P_{b}\right)$ is the oxyhemoglobin saturation, $a_{b}$ is the solubility of oxygen in blood, and $P_{b}$ is the blood $\mathrm{PO}_{2}[16,17]$. Given the source strengths, the resulting oxygen concentration at a tissue point is calculated by summing the oxygen fields (called Green's functions) produced by each of the surrounding blood vessels. It is important to note that the Green's function approach, which has been implemented in other applications [18, 31], is especially useful since it allows for more efficient computation and is amenable to parallelization.

\subsection{Model simulations}

In this study, blood flow and pressure are predicted throughout the entire retinal microcirculation. In addition, the arteriolar blood oxygen saturation levels are predicted 
within the realistic network geometry of the mouse retina using the Green's function approach. When modeling the arteriolar network in isolation, the blood pressure entering the arterial network is assumed to be $40 \mathrm{mmHg}$ (based on outgoing pressure measures of the central retinal artery), and the pressure exiting the arterioles is assumed to be $24 \mathrm{mmHg}$ (this value is chosen to generate a pressure drop across the arterioles that is consistent with that predicted by previous models [34]). The incoming blood to the retinal circulation is assumed to be well oxygenated with a $\mathrm{PO}_{2}$ of 84.4 $\mathrm{mmHg}$ (saturation of $96 \%$ ). Flow to the arteriolar network is set at $6.17 \times 10^{-6} \mathrm{~cm}^{3} / \mathrm{s}$, which is consistent with the value of flow used by Ganesan et al. [15] and gives an overall pressure drop of $25 \mathrm{mmHg}$ across the entire system. The value of oxygen demand $\left(M_{0}\right.$ in Eq. 7$)$ is varied from low to high levels $\left(M_{0}=1\right.$ to $M_{0}=8 \mathrm{~cm}^{3} \mathrm{O}_{2} / 100$ $\mathrm{cm}^{3} / \mathrm{min}$ ), and the model is used to predict the resulting blood and tissue oxygen distributions in the arteriolar circulation. The mean and standard deviations of arteriolar outflow $\mathrm{PO}_{2}$ values are also compared.

\section{Results}

Given the flow and pressure assumptions listed in Section 2.4, Figure 4 shows the predicted distribution of flows and diameters of the arteriolar and venular vessels in the retinal network, plotted on a log-log scale. A power-law best-fit was made to the flowdiameter data $\left(Q \sim D^{k}\right)$, giving a $k$ value of 2.9936 for the arteriolar vessels and 2.8920 for the venular vessels. This best-fit (solid red line) was compared with the best-fit power-law relationship for the flows predicted by Ganesan et al. [15] (dashed blue line). The values of $k$ obtained by Ganesan et al were $k=2.9496$ and 2.9508 for the arterial and venous networks, respectively. While the slopes of these best-fit lines are nearly identical, the intercepts have a larger discrepancy due to the larger assumed pressure drop across the network in the Ganesan et al. study. Ganesan et al. assumed a pressure drop of $40 \mathrm{mmHg}$ from the incoming retinal artery to outgoing retinal vein, while the current study assumes a pressure drop of $25 \mathrm{mmHg}$ since the outgoing pressure is assumed to be $15 \mathrm{mmHg}$, not $0 \mathrm{mmHg}$, to prevent venous collapse due to typical values of IOP.

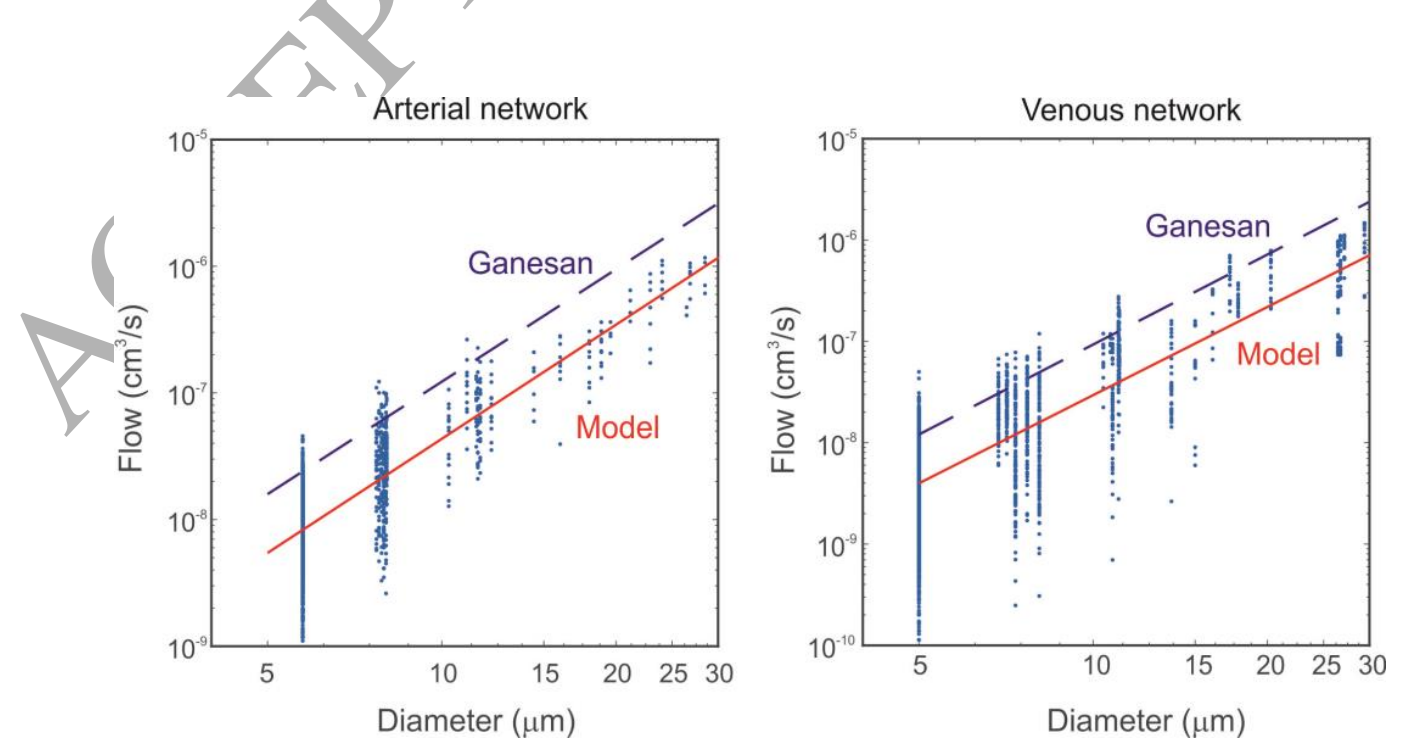


Figure 4. Log-log plot of flow $\left(\mathrm{cm}^{3} / \mathrm{s}\right)$ versus diameter $(\mu \mathrm{m})$ in each of the arterial vessels (left panel) and each of the venous vessels (right panel). Dotted blue line (--) represents the best-fit power-law line from the Ganesan et al. 2010 [15] study; solid red line (-) represents the best-fit power-law line from the current study.

Figure 5 shows the average intravascular pressure in arterioles, capillaries, and venules predicted using the current model (red stars) and Ganesan et al. study (blue squares). In the arterial network, the pressures drop from an average of $39.28 \mathrm{mmHg}$ in the (largest) order 5 vessels down to an average of $32.82 \mathrm{mmHg}$ in the (smallest) order 1 vessels. In the venous network, the pressures drop from an average of $24.16 \mathrm{mmHg}$ in the (smallest) order 1 vessels down to an average of $16.24 \mathrm{mmHg}$ in the (largest) order 5 vessels. The figure does not show a similar decrease in the capillaries because the horizontal axis is not vessel branching order, as in the arterial and venous subfigures. Rather, the pressures are predicted at different radial distances from the center of the capillary rings, and vessels further away from the center are not necessarily of higher or lower order.
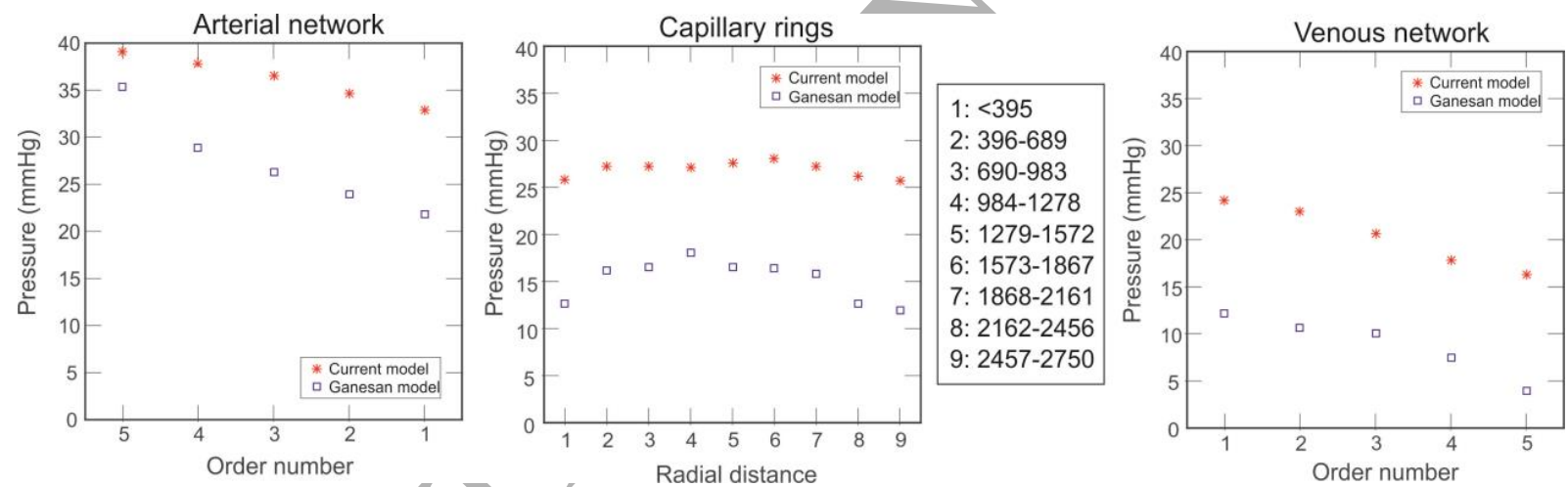

Figure 5. Plot of average pressure $(\mathrm{mmHg}$ ) versus order number (or radial distance for capillaries) in the arterioles (left), capillary ring vessels (center), and venules (right). Red stars $\left(^{*}\right)$ represent the predicted pressure averages from the current study; blue squares ( $\square$ ) represent the predicted pressure averages from the Ganesan et al. [15] study.

Figure 6 shows predicted oxygen levels throughout the arteriolar network as tissue oxygen demand $\left(M_{0}\right)$ is varied in the four panels $\left(1,2,4\right.$, and $\left.8 \mathrm{~cm}^{3} \mathrm{O}_{2} / 100 \mathrm{~cm}^{3} / \mathrm{min}\right)$. The model predicts that the tissue $\mathrm{PO}_{2}$ drops noticeably as oxygen demand is increased, indicated by larger areas of blue in the contour plots. 
$\mathrm{M}_{0}=1$

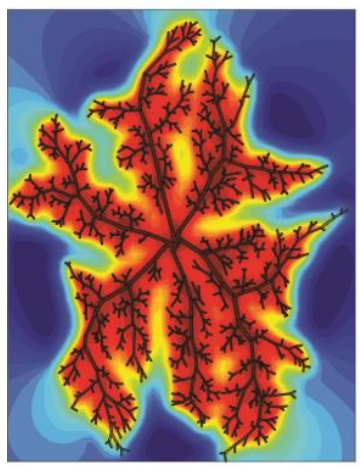

$-0.2 \mathrm{~mm}$
$\mathrm{M}_{0}=2$

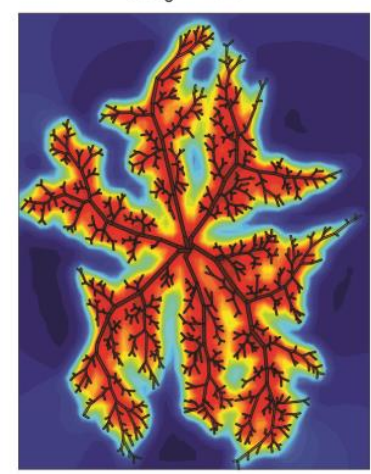

$\mathrm{M}_{0}=4$

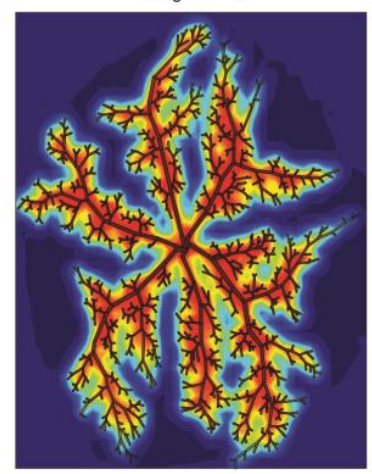

$\mathrm{M}_{0}=8$

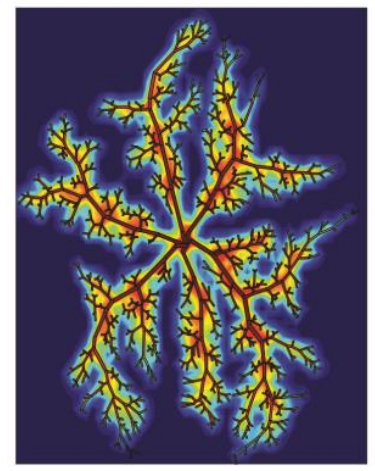

Figure 6. Contour plot of predicted oxygen levels in the arteriolar network and in the surrounding tissue for oxygen demand values of $1,2,4$, and $8 \mathrm{~cm}^{3} \mathrm{O}_{2} / 100 \mathrm{~cm}^{3} / \mathrm{min}$. Red indicates areas of high oxygenation, and blue indicates areas of low oxygenation (color scale provided, in $\mathrm{mmHg}$ ).

To quantify the changes in $\mathrm{PO}_{2}$ as oxygen demand is increased, Figure 7 (left) shows the average $\mathrm{PO}_{2}$ of the arteriolar outflow vessels as $\mathrm{M}_{0}$ is varied. As $\mathrm{M}_{0}$ increases, the average outflow arteriolar vessel $\mathrm{PO}_{2}$ is predicted to decrease from 80.60 to 71.65 $\mathrm{mmHg}$. Because the outflowing arterioles in the heterogeneous network create different pathways for blood flow into capillaries, the distribution of $\mathrm{PO}_{2}$ in these vessels is quantified, as well. Figure 7 (right) shows the standard deviation of arteriolar vessel $\mathrm{PO}_{2}$ with increasing oxygen demand. Unlike the average vessel $\mathrm{PO}_{2}$, the standard deviation of vessel $\mathrm{PO}_{2}$ increases from 6.39 to 12.69 as $\mathrm{M}_{0}$ is increased from 1 to $8 \mathrm{~cm}^{3}$ $\mathrm{O}_{2} / 100 \mathrm{~cm}^{3} / \mathrm{min}$, indicating that the spread in $\mathrm{PO}_{2}$ is widening as the mean $\mathrm{PO}_{2}$ is decreasing.
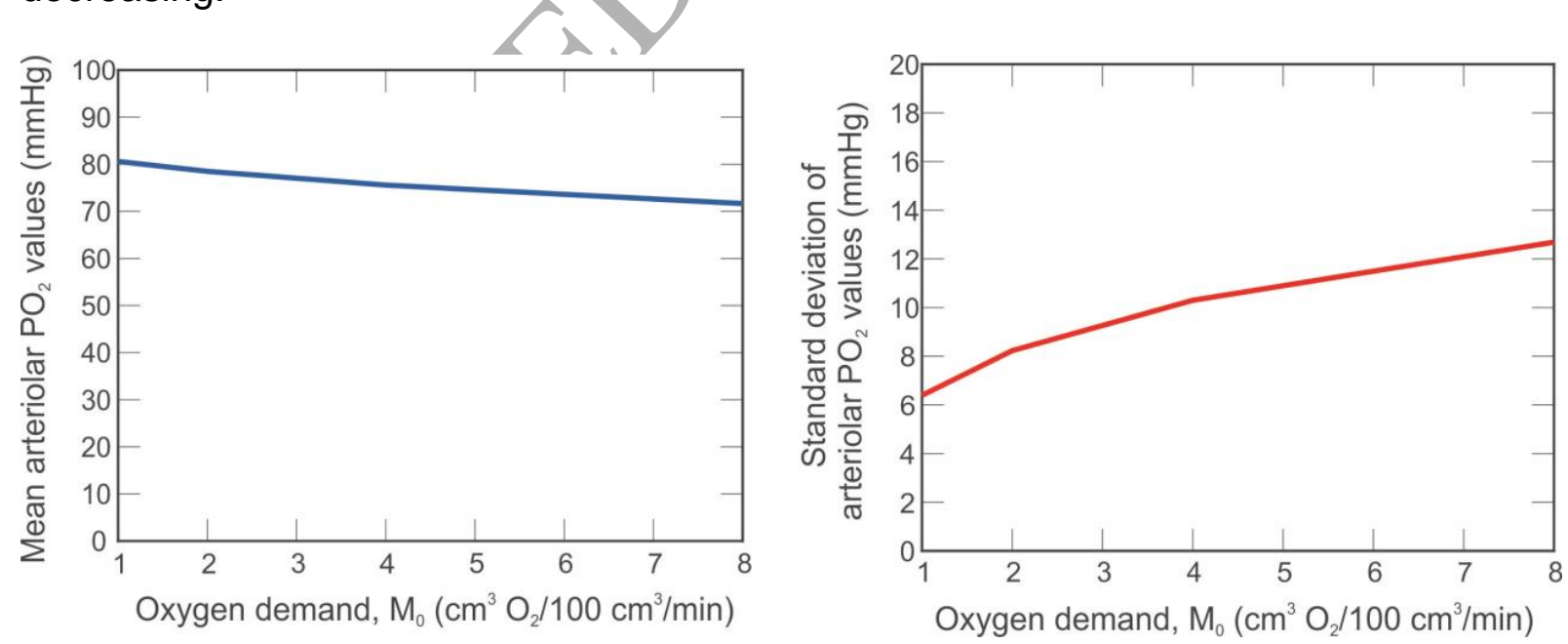

Figure 7. Left: Plot of mean arterial vessel $\mathrm{PO}_{2}$ (in $\mathrm{mmHg}$ ) versus oxygen demand (in $\mathrm{cm}^{3} \mathrm{O}_{2} / 100 \mathrm{~cm}^{3} / \mathrm{min}$ ). Right: Plot of standard deviation of arterial vessel $\mathrm{PO}_{2}$ versus oxygen demand. 


\section{Discussion}

The complex retinal vascular network modeled in this study is based on a vast amount of previously-collected experimental data $[14,15]$ and is solved using non-trivial and highly powerful mathematical tools. The model is used to predict blood flow and pressure in each of the microvessels of the entire network, as well as the $\mathrm{PO}_{2}$ in the arteriolar network and surrounding tissue.

Model results predict a steady drop in pressure throughout the network, with an average pressure in the largest arterioles of $39.28 \mathrm{mmHg}$ and an average pressure of 16.24 $\mathrm{mmHg}$ in the largest venules. Predicted blood flow rates in the Ganesan et al. study were much larger than in the current study (Figure 4), but this is due to differing assumptions in the overall pressure drop along the entire network in this study and the Ganesan et al. study. Nevertheless, the power-law relationship between blood flow rate and diameter of the vessels, $Q \sim D^{k}$, in the arteriolar and venular networks predicted in the two studies is very similar. The current study predicts $k$ values of 2.9936 and 2.8920 for the arteriolar and venular networks, respectively, as compared with values 2.9496 and 2.9508 in the Ganesan et al. study. This similarity indicates that the blood flow rates in the retinal microcirculation scale similarly with diameter regardless of the chosen pressure drop. This scaling is also consistent with power law relationships between flow and diameter in arterial and venous networks obtained by a mathematical model by Causin et al. [11] and experimental findings by Riva et al. [35], which found $k$ values of 2.76 and 2.84 for the two networks, respectively.

The model is also used to predict $\mathrm{PO}_{2}$ levels in the arteriolar vessels and surrounding tissue. Although the $\mathrm{PO}_{2}$ values in the arteriolar network do not take into account the intermediate (or deep) layer of the full network, the diffusion distance of oxygen in tissue is very short and thus vessels in the intermediate (or deep) layer are too far from the superficial layer to have much, if any, effect on the oxygenation of the arteriolar network. Model results indicate varying distributions of oxygen levels depending on the assumed tissue oxygen demand. With increasing levels of oxygen demand from 1 to $8 \mathrm{~cm}^{3}$ $\mathrm{O}_{2} / 100 \mathrm{~cm}^{3} / \mathrm{min}$, average arteriolar $\mathrm{PO}_{2}$ is predicted to drop by $8.95 \mathrm{mmHg}$, from 80.60 to $71.65 \mathrm{mmHg}$. These predictions are within range of previous modeling studies by Causin et al. [11] and Liu et al. [10]. Causin et al. [11] predicted an average arteriolar $\mathrm{PO}_{2}$ between 49 and $77 \mathrm{mmHg}$ for an oxygen demand of $3.6 \mathrm{~cm}^{3} \mathrm{O}_{2} / 100 \mathrm{~cm}^{3} / \mathrm{min}$, and Liu et al. [10] predicted $\mathrm{PO}_{2}$ values between 65 and $108 \mathrm{mmHg}$ in an arteriolar network.

In the current/study, model simulations in the heterogeneous arteriolar network show a wide spread of $\mathrm{PO}_{2}$ values in the network. In particular, as oxygen demand is increased from 1 to $8 \mathrm{~cm}^{3} \mathrm{O}_{2} / 100 \mathrm{~cm}^{3} / \mathrm{min}$, the standard deviation of vessel $\mathrm{PO}_{2}$ nearly doubles, from 6.39 to $12.69 \mathrm{mmHg}$. This increase in standard deviation indicates that the spread in $\mathrm{PO}_{2}$ is widening and that the average value of $\mathrm{PO}_{2}$ is a poor indicator of network oxygenation. This is of particular concern because the heterogeneity of the network leads to hundreds of non-equivalent pathways from the inflow vessel to an outflow arteriole; each of these outflow arterioles delivers blood downstream to the capillaries, where the majority of oxygen consumption takes place. With high oxygen demand, the 
$\mathrm{PO}_{2}$ distribution in the arteriolar network is shifted to lower values, and the tail of the distribution is larger, indicating that some terminal arteriolar vessels will have abnormally low $\mathrm{PO}_{2}$ levels. This can create areas of poor oxygenation at risk of hypoxia downstream, an effect that would not be observed in a non-heterogeneous description of the network, or if the average vessel $\mathrm{PO}_{2}$ was the sole indicator of oxygenation. Thus, the techniques proposed in this study provide a more realistic modeling approach for predicting regions of hypoxia as would occur in glaucomatous patients, since decreased oxygenation of retinal ganglion cells causes damage to these cells, lack of function, and eventually apoptosis and irreversible blindness [36]. Mechanistically, regional hypoxia has been shown to disrupt axonal transport disruption in rat models [37]. Patients with advanced glaucoma have higher oxygen saturation in venules and lower arteriovenous difference in oxygen saturation compared with healthy individuals [38] while variability of venous oxygen saturation has also been identified in other eye diseases such as in central retinal vein occlusion [39].

Model limitations. Unlike the arteriolar and venular components of the retinal network, which were obtained from actual microscopy images in the mouse retina, the capillary network was created using the rule-based algorithm proposed by Ganesan et al. [15]. Thus, the capillary network comprises $82 \%$ of the vessels in the entire network and yet is the least realistic component of the retinal network used in this study. As a result, predictions of $\mathrm{PO}_{2}$ in the capillary network were not provided here since any conclusions about the distribution of oxygen in the capillary network could potentially be an artifact of the algorithm used to create the network. In addition, although the Green's function method is relatively efficient compared to other methods, implementing the current version of the capillary network in the oxygenation calculation leads to an unreasonable computational time using this method. For those reasons, the model presented in this work will be altered in future studies to include a simplified capillary compartment. Such a simplification will not result in a loss of heterogeneous information about the retinal vasculature and will allow for more computational time to be spent simulating blood flow regulation in the arterioles.

It is important to note that the network defined in this study is based on measurements from mouse retina. If the same network geometry and morphology information becomes available in the human eye, then it would be a straight-forward exercise to adapt this model to the human retina (which contains 4 , not 6 , main arterial branches) since the model algorithms and calculations would be the same. Nevertheless, similar results in flow and oxygenation are expected for healthy human eyes.

Concluding remarks.

In this study, blood flow and oxygenation were predicted in a retinal vascular network at steady state. However, arteriolar vessels have the ability to constrict or dilate actively and passively in response to various stimuli - a process known as blood flow regulation. Impaired blood flow regulation has been implicated as a possible contributing factor to the abnormal oxygenation observed in glaucoma. Thus, to predict changes in oxygenation in glaucoma patients, this Green's function model will be adapted to include mechanisms of blood flow regulation that have been described in a previous 
compartmental modeling approach [28]. Inclusion of regulation will improve the predictive power of this model and eventually provide insight into the relationship between tissue oxygenation and retinal function, allowing theoretical approaches to address the controversy of the cause-and-effect relationship between retinal ganglion cell death and impaired retinal blood flow in glaucoma patients. Such work has the potential to improve diagnostic and treatment strategies for the millions of glaucoma patients throughout the world.

\section{Acknowledgments}

$\mathrm{AH}$ and BS acknowledge Research to Prevent Blindness.

\section{Funding}

BF gratefully acknowledges Burroughs Wellcome Fund Collaborative Research Travel Grant 1017517. JA gratefully acknowledges NSF DMS-1654019.

\section{Author Disclosure Statement}

There are no conflicts of interest. Dr. Alon Harris would like to disclose that he receives remuneration from CIPLA and AdOM for serving as a consultant. Dr. Harris also holds an ownership interest in AdOM, Nano Retina, and Oxymap. All relationships listed above are pursuant to Indiana University's policy on outside activities.

\section{References}

1. Anderson, D.R., Normal-tension glaucoma (Low-tension glaucoma). Indian J Ophthalmol, 2011. 59 Suppl: p. S97-101.

2. Potop, V., M. Dumitrache, and A. Ciocalteu, [Normal tension glaucoma]. Oftalmologia, 2010. 54(2): p. 11-4.

3. Harris, A., et al., Measuring and interpreting ocular blood flow and metabolism in glaucoma. Can J Ophthalmol, 2008. 43(3): p. 328-36.

4. Hayreh, S.S., Blood flow in the optic nerve head and factors that may influence it. Prog Retin Eye Res, 2001. 20(5): p. 595-624.

5. Leske, M.C., et al., Predictors of long-term progression in the early manifest glaucoma trial. Ophthalmology, 2007. 114(11): p. 1965-72.

6. Moore, D., et al., Dysfunctional regulation of ocular blood flow: A risk factor for glaucoma? Clin Ophthalmol, 2008. 2(4): p. 849-61.

7. Olafsdottir, O.B., et al., Retinal oxygen metabolism in healthy subjects and glaucoma patients. British Journal of Ophthalmology, 2014. 98(3): p. 329-333.

8. Carichino, L., et al., A theoretical investigation of the increase in venous oxygen saturation levels in glaucoma patients. Investigative Ophthalmology \& Visual Science, 2015. 56(7).

9. Bernabeu, M.O., et al., Computer simulations reveal complex distribution of haemodynamic forces in a mouse retina model of angiogenesis. J R Soc Interface, 2014. 11(99). 
10. Liu, D., et al., Computational analysis of oxygen transport in the retinal arterial network. Curr Eye Res, 2009. 34(11): p. 945-56.

11. Causin, P., et al., Blood flow mechanics and oxygen transport and delivery in the retinal microcirculation: multiscale mathematical modeling and numerical simulation. Biomech Model Mechanobiol, 2016. 15(3): p. 525-42.

12. Malek, J., et al., Computational analysis of blood flow in the retinal arteries and veins using fundus image. Computers \& Mathematics with Applications, 2015. 69(2): p. 101-116.

13. Malek, J., A.T. Azar, and R. Tourki, Impact of retinal vascular tortuosity on retinal circulation. Neural Computing \& Applications, 2015. 26(1): p. 25-40.

14. Ganesan, P., S. He, and H. Xu, Development of an image-based model for capillary vasculature of retina. Comput Methods Programs Biomed, 2011. 102(1): p. 35-46.

15. Ganesan, P., S. He, and H. Xu, Development of an image-based network model of retinal vasculature. Ann Biomed Eng, 2010. 38(4): p. 1566-85.

16. Secomb, T.W., A Green's function method for simulation of time-dependent solute transport and reaction in realistic microvascular geometries. Math Med Biol, 2016. 33(4): p. 475-494.

17. Secomb, T.W., et al., Green's function methods for analysis of oxygen delivery to tissue by microvascular networks. Ann Biomed Eng, 2004. 32(11): p. 1519-29.

18. Fry, B.C., T.K. Roy, and T.W. Secomb, Capillary recruitment in a theoretical model for blood flow regulation in heterogeneous microvessel networks. Physiol Rep, 2013. 1(3): p. e00050.

19. Fry, B.C., et al., Estimation of Blood Flow Rates in Large Microvascular Networks. Microcirculation, 2012. 19(6): p. 530-538.

20. Lee, J., et al., Automatic segmentation of 3D micro-CT coronary vascular images. Med Image Anal, 2007. 11(6): p. 630-47.

21. Sinclair, M.D., et al., Measurement and modeling of coronary blood flow. Wiley Interdiscip Rev Syst Biol Med, 2015. 7(6): p. 335-56.

22. Lee, J. and N.P. Smith, The multi-scale modelling of coronary blood flow. Ann Biomed Eng, 2012. 40(11): p. 2399-413.

23. Gagnon, L., et al., Modeling of Cerebral Oxygen Transport Based on In vivo Microscopic Imaging of Microvascular Network Structure, Blood Flow, and Oxygenation. Front Comput Neurosci, 2016. 10: p. 82.

24. Willermain, F., et al., Origins and consequences of hyperosrnolar stress in retinal pigmented epithelial cells. Frontiers in Physiology, 2014. 5.

25. Pries, A.R., Secomb, T.W., Blood flow in microvascular networks., in Handbook of Physiology: Section 2, The Cardiovascular System, vol. IV, Mircrocirculation, R.F. Tuma, Duran W. N., Ley K., Editor. 2008, Academic Press: San Diego, CA. p. 3-36.

26. Pries, A.R., et al., Blood flow in microvascular networks. Experiments and simulation. Circ Res, 1990. 67(4): p. 826-34.

27. Young, D.M., Iterative methods for solving partial difference equations of elliptic type. Transactions of the American Mathematical Society, 1954. 76: p. 92-111.

28. Arciero, J., et al., Theoretical analysis of vascular regulatory mechanisms contributing to retinal blood flow autoregulation. Invest Ophthalmol Vis Sci, 2013. 54(8): p. 5584-93.

29. Arciero, J.C., B.E. Carlson, and T.W. Secomb, Theoretical model of metabolic blood flow regulation: roles of ATP release by red blood cells and conducted responses. Am J Physiol Heart Circ Physiol, 2008. 295(4): p. H1562-71.

30. Carlson, B.E., J.C. Arciero, and T.W. Secomb, Theoretical model of blood flow autoregulation: roles of myogenic, shear-dependent, and metabolic responses. Am J Physiol Heart Circ Physiol, 2008. 295(4): p. H1572-9. 
31. Roy, T.K., A.R. Pries, and T.W. Secomb, Theoretical comparison of wall-derived and erythrocytederived mechanisms for metabolic flow regulation in heterogeneous microvascular networks. Am J Physiol Heart Circ Physiol, 2012. 302(10): p. H1945-52.

32. McGuire, B.J. and T.W. Secomb, A theoretical model for oxygen transport in skeletal muscle under conditions of high oxygen demand. J Appl Physiol (1985), 2001. 91(5): p. 2255-65.

33. Krogh, A., The number and distribution of capillaries in muscles with calculations of the oxygen pressure head necessary for supplying the tissue. J Physiol, 1919. 52(6): p. 409-15.

34. Guidoboni, G., et al., Intraocular pressure, blood pressure, and retinal blood flow autoregulation: a mathematical model to clarify their relationship and clinical relevance. Invest Ophthalmol Vis Sci, 2014. 55(7): p. 4105-18.

35. Riva, C.E., et al., Blood velocity and volumetric flow rate in human retinal vessels. Invest Ophthalmol Vis Sci, 1985. 26(8): p. 1124-32.

36. Evangelho, K., et al., Pathophysiology of primary open-angle glaucoma from a neuroinflammatory and neurotoxicity perspective: a review of the literature. Int Ophthalmol, 2017.

37. Chidlow, G., J.P.M. Wood, and R.J. Casson, Investigations into Hypoxia and Oxidative Stress at the Optic Nerve Head in a Rat Model of Glaucoma. Front Neurosci, 2017. 11: p. 478.

38. Olafsdottir, O.B., et al., Retinal oxygen metabolism in healthy subjects and glaucoma patients. $\mathrm{Br}$ J Ophthalmol, 2014. 98(3): p. 329-33.

39. Eliasdottir, T.S., et al., Venous oxygen saturation is reduced and variable in central retinal vein occlusion. Graefes Arch Clin Exp Ophthalmol, 2015. 253(8): p. 1409. 